\title{
Comunicação conjugal negativa e aberta: modelo interdependente de efeito ator/parceiro no ajustamento conjugal
}

\section{Negative and open marital communication: interdependent model of actor/partner effect in marital adjustment}

\section{Comunicación conyugal negativa y abierta: modelo interdependiente de efecto actor- compañero en el ajuste conyugal}

Crístofer Batista da Costa ${ }^{1}$ ORCID 0000-0001-8914-3144

Clarisse Pereira Mosmann ${ }^{2}$ ORCID 0000-0002-9275-1105

\author{
12 Programa de Pós-Graduação em Psicologia Clínica, Centro de Ciências da Saúde, \\ Universidade do Vale do Rio dos Sinos- UNISINOS-Brasil
}

\begin{abstract}
Resumo: A comunicação é imprescindível à avaliação e intervenção em psicoterapia conjugal. Nesse sentido, foi investigado se a comunicação conjugal negativa e aberta impactam no ajustamento conjugal de casais heterossexuais. Trata-se de um estudo quantitativo, transversal, correlacional e explicativo. Foram avaliados 231 casais residentes no Sul do Brasil. Os participantes responderam o Communication Questionnaire e o Revised Dyadic Adjustment Scale R-DAS. Os dados foram analisados de forma diádica por meio do Actor Partner Interdependence Model - APIM e indicaram que a comunicação negativa provoca impacto superior à comunicação aberta no ajustamento conjugal de maridos e esposas, já que provocou efeitos ator e parceiro. A comunicação aberta provocou somente o efeito ator para maridos e esposas. Os resultados foram discutidos à luz da literatura científica considerando as possíveis implicações para a área clínica e para a pesquisa.
\end{abstract}

Palavras-chave: comunicação; relacionamento conjugal; ajustamento conjugal

Abstract: Communication is essential for assessment and intervention in marital psychotherapy. For that matter, it was investigated whether negative and open marital communication impact the marital adjustment of heterosexual couples. This is a quantitative, cross-sectional, correlational, and explanatory study. A total of 231 couples residing in the Southern region of Brazil were assessed. Participants answered the Communication Questionnaire and the Revised Dyadic Adjustment Scale (R-DAS). The data were analyzed on a dyadic basis using the Actor Partner Interdependence Model (APIM) and indicated that negative communication causes a greater impact than open communication in the marital adjustment of husbands and wives, since it caused actor and partner effects. Open communication caused only the actor effect for husbands and wives. The results were discussed in the light of the scientific literature considering the possible implications for the clinical area and for the research.

Keywords: communication; marital relationship; marital adjustment 
Resumen: La comunicación es indispensable para la evaluación e intervención en psicoterapia conyugal. En ese sentido, fue investigado si los estilos de comunicación conyugal negativa y abierta impactan en el ajuste conyugal de parejas heterosexuales. Se trata de un estudio cuantitativo, transversal, correlacional y explicativo. Se evaluaron 231 parejas residentes en el Sur de Brasil. Los participantes respondieron el Communication Questionnaire y el Revised Dyadic Adjustment Scale R-DAS. Los datos fueron analizados de forma diádica a través del Actor Partner Interdependence Model - APIM e indicaron que la comunicación negativa produce un impacto superior a la comunicación abierta en el ajuste conyugal de maridos y esposas, pues provocó los efectos actor y socio. La comunicación abierta fue predictora solamente del efecto actor para los esposos y las esposas. Los resultados fueron discutidos a la luz de la literatura científica y en consideración de las posibles implicaciones para el área clínica y para la investigación.

Palabras clave: comunicación; relación conyugal; ajuste conyugal

Recebido: 27/03/2019

Aceito: 01/09/2020

Como citar:

Costa, C.B., \& Mosmann, C.P. (2020). Comunicação conjugal negativa e aberta: modelo interdependente de efeito ator/parceiro no ajustamento conjugal. Ciencias Psicológicas, 14(2), e-2283. doi: https://doi.org/10.22235/cp.v14i2.2283

Correspondência: Crístofer Batista da Costa. Rua Marcelo Gama, 1440/304, Auxiliadora. CEP 90540-041. Porto Alegre, RS, Brasil. E-mail: cristoferbatistadacosta@gmail.com. Clarisse Pereira Mosmann. Av. Unisinos, 950 Sala 2a109, Jardim Itu Sabará. CEP 93022000. São Leopoldo, RS, Brasil. E-mail: clarissemosmann@ gmail.com

\section{Introdução}

A comunicação é imprescindível à existência humana e consensualmente relevante em psicoterapia de casal, conforme aponta revisão sistemática da literatura nacional e internacional (Costa, Delatorre, Wagner, \& Mosmann, 2017). Os programas de educação conjugal (Blanchard, Hawkins, Baldwin, \& Fawcett, 2009; Epstein, Warfel, Johnson, Smith, \& McKinney, 2013; Lau, Tao, Randall, \& Bodenmann, 2016; Neumann \& Wagner, 2017; Neumann, Wagner, \& Remor, 2018) e de avaliação de processos e resultados em psicoterapia de casal (Baucom, Baucom, \& Christensen, 2015; Tilden, Hoffart, Sexton, Finset, \& Gude, 2011; Worthington Jr. et al., 2015), visando melhorar, entre outros aspectos, as habilidades de comunicação dos parceiros, têm apontado evidências consistentes dos efeitos positivos das intervenções, promovendo aumentos nos níveis de ajustamento conjugal.

A análise da comunicação nas relações humanas e os pressupostos de circularidade e de retroalimentação surgiram inicialmente na Escola de Palo Alto (EUA) por meio das contribuições de autores como Gregory Bateson, Paul Watzlawick, Janet Beavin, Don Jackson, Jay Haley, John Wekland, entre outros, pautados essencialmente em uma abordagem sistêmica (Boscolo \& Bertrando, 2013; Mattelart, 2009). Segundo esses autores, o emissor e o receptor da mensagem têm papel igualmente importante no curso da interação (Mattelart, 2009; Watzlawick, Beavin, \& Jackson, 1973). Nessa perspectiva, os processos relacionais, interacionais e o contexto em que ocorre a troca de mensagens deve ser o aspecto principal de análise em detrimento da observação de variáveis individuais isoladamente (Costa, Cenci, \& Mosmann, 2016; Féres-Carneiro \& DinizNeto, 2010). 
Mesmo que um indivíduo tenha a intenção de não se expressar verbalmente, ele estará comunicando algo, preceito de que é impossível não comunicar. O relato (informação, conteúdo) e o comando (ordem, conotação) são os principais aspectos que constituem a comunicação. $\mathrm{O}$ primeiro se refere à transmissão de informação, dados etc., e o segundo, considerado metacomunicação, está relacionado à intenção, propósito subjacente, interferindo diretamente em como a mensagem será recebida (Watzlawick et al., 1973). Segundo os autores, se a relação entre os cônjuges for harmoniosa, a comunicação se torna um aspecto secundário. Entretanto, se for conflituosa, mais esforço precisará ser despendido para que o conteúdo fique evidente. Portanto, as características da comunicação entre o casal são relevantes tanto para a avaliação da dinâmica conjugal como para a intervenção em contexto clínico (Baucom et al., 2015; Tilden et al., 2011; Worthington Jr. et al., 2015).

A comunicação, se for negativa, ocorrerá por meio de comportamentos de acusação, dominância, interrupções, retraimento, indiferença, tom de voz hostil e um contexto de interações negativas que tendem a se agravar, provocando o conflito em escalada. Sendo positiva, a comunicação ocorre por meio de empatia, compreensão, validação, abertura para falar de forma clara e sincera sobre problemas e interesses de ordem pessoal, conjugal, profissional, entre outros (Van den Troost, Vermulst, Gerris, \& Matthijs, 2005).

Além disso, a objetividade e a clareza, a capacidade de ouvir, compartilhar pensamentos e sentimentos honestamente e evitar fazer críticas são características de uma comunicação conjugal positiva e competências para uma relação satisfatória (Epstein et al., 2013). Para testar esse argumento, Epstein e colaboradores avaliaram sete fatores considerados competências importantes em um relacionamento romântico em uma amostra de 2.201 participantes. Os fatores avaliados foram: comunicação, resolução de conflito, conhecimento do parceiro, habilidades para a vida, gestão de si, sexo/intimidade e gestão do estresse. Os resultados do estudo confirmaram que os programas de educação conjugal são benéficos aos casais, já que os participantes dos programas de treinamento em comunicação e resolução de conflito pontuaram mais nas competências avaliadas. Na mesma direção em que apontam outras pesquisas (Baucom et al., 2015; Johnson et al., 2005; Markman, Rhoades, Stanley, Ragan, \& Whitton, 2010; Tilden et al., 2011; Worthington Jr. et al., 2015), o referido estudo indicou que as habilidades de comunicação são preditoras de maiores níveis de satisfação e ajustamento conjugal.

No estudo de Markman et al. (2010) a qualidade da comunicação antes do casamento e durante os primeiros cinco anos de união foi avaliada em relação ao conflito e ao divórcio em uma amostra de 210 casais norteamericanos. Os resultados confirmaram o que os autores denominaram de "efeito da negatividade", em que a comunicação negativa é fator de risco mais forte do que a comunicação positiva é fator de proteção, conforme encontrado também por Johnson et al. (2005). A comunicação negativa esteve fortemente associada ao declínio na qualidade e no ajustamento conjugal e ao risco de divórcio. Os autores explicam que esse resultado pode indicar que as pessoas são mais sensíveis às experiências negativas no relacionamento e na vida, já que elas podem efetivamente machucar mais e, portanto, são selecionadas e mantidas sob atenção em detrimento dos aspectos positivos.

Além disso, os resultados do estudo de Markman et al. (2010) apoiam o pressuposto de que é necessário atentar para os fatores pré-interacionais, observando o momento adequado para conversar sobre os conflitos (Costa \& Mosmann, 2015), e substituir expressões negativas de raiva e crítica por expressões de afeto e admiração que repercutem na interação conjugal em longo prazo (Féres-Carneiro \& Diniz-Neto, 2010) e promovem cooperação entre a dupla (Paleari, Regalia, \& Fincham, 2010). Outros estudos apontam ainda que a comunicação conciliatória contribui para a superação de possíveis dificuldades inerentes as diferentes etapas do ciclo vital conjugal (Luz \& Mosmann, 2018; Rech, Silva, \& Lopes, 2013).

Uma pesquisa conduzida por meio de questionário online com uma amostra de 266 pessoas em relações heterossexuais estáveis no Rio Grande do Sul, Brasil, avaliou e comparou a 
comunicação de indivíduos com filhos, em distintas etapas do ciclo vital familiar e com diferentes níveis de funcionalidade medidos por meio da coesão, da adaptabilidade e do ajustamento conjugal. Os resultados demonstraram que a comunicação negativa é um aspecto que perpassa diferentes etapas do casamento, especialmente os anos iniciais. Indicou ainda que os homens utilizaram mais o estilo de comunicação negativa e as mulheres o estilo de comunicação aberta (Luz \& Mosmann, 2018).

Em Berkeley, na Califórnia (EUA), Seider, Hirschberger, Nelson e Levenson (2009) avaliaram a comunicação e os padrões de interação conjugal por meio dos pronomes de tratamento eu, tu e nós, em uma pesquisa que analisou quinze minutos de conversa de 154 casais e mediu a qualidade da interação, do afeto e a satisfação conjugal. Os resultados forneceram evidências de que a utilização do pronome "nós" reflete interdependência, responsabilidade compartilhada, parceria e resolução construtiva dos conflitos e associação com altos níveis de afeto positivo, baixos níveis de afeto negativo e de excitação cardiovascular, sendo mais fortes ao receptor da mensagem - efeito parceiro. Os pronomes de separação "eu" e "tu" refletiram afastamento, individualismo, altos níveis de afeto e interação negativa e insatisfação conjugal, efeitos substanciais a ambos, ator e parceiro. Os autores concordam que os casais satisfeitos utilizam com mais frequência tentativas de reparação que impedem ou reduzem a negatividade durante as discussões, aceitam a influência do cônjuge e a existência de problemas insolúveis e são solícitos ao parceiro validando sua tentativa de interação (Costa \& Mosmann, 2015; Driver, Tabares, Shapiro, \& Gottman, 2016; Madhyastha, Hamaker, \& Gottman, 2011).

Na mesma direção investigativa, Madhyastha et al. (2011) analisaram quinze minutos de interação de 254 casais, em intervalos de 6 segundos, avaliando o quanto o estado de equilíbrio emocional de um cônjuge era capaz de influenciar o do parceiro com base no intervalo anterior. Casais cujos níveis de satisfação conjugal eram baixos permaneciam em um contexto de reciprocidade negativa por mais tempo, e aqueles com maiores níveis de satisfação estabeleciam um clima de acordo e de maior positividade durante o conflito, mais aprovação e menos divergências e críticas. Os autores constataram a influência recíproca entre os parceiros, aspecto que Driver et al. (2016) apontam como necessário em um relacionamento conjugal, embora a interação deva ocorrer por meio de aproximadamente cinco comportamentos positivos para cada comportamento negativo. O referido padrão foi proposto a partir de extensa pesquisa conduzida pelos investigadores do laboratório Gottman e diferencia casais satisfeitos de casais insatisfeitos e divorciados.

Os pressupostos teóricos e empíricos apresentados evidenciam a relevância da comunicação no relacionamento conjugal. Trata-se de um aspecto que possibilita observar as características da interação entre os cônjuges (Costa et al., 2016; Féres-Carneiro \& Diniz-Neto, 2010), e no qual os casais podem investir no sentido de prevenir problemas futuros, participando, por exemplo, de programas de educação conjugal pré e pós núpcias (Blanchard et al., 2009; Epstein et al., 2013; Lau et al., 2016; Neumann \& Wagner, 2017; Neumann et al., 2018). Ademais, nas psicoterapias de casal baseadas em evidências, a comunicação entre os cônjuges aparece nos estudos como um dos principais focos da intervenção, promotores de resultados positivos (Baucom et al., 2015; Costa et al., 2017; Tilden et al., 2011; Worthington Jr. et al., 2015).

Os estudos sobre comunicação têm utilizado, principalmente, o ajustamento conjugal como variável de desfecho (Belanger, Laporte, Sabourin, \& Wright, 2015; Driver et al., 2016; Holley, Haase, \& Levenson, 2013; Worthington Jr. et al., 2015), dimensão do relacionamento que envolve coesão, consenso e satisfação conjugal (Hollist et al., 2012). No contexto clínico, resultados de estudos dessa natureza poderão orientar intervenções com vistas a aumentar os níveis de ajustamento conjugal (Madhyastha et al., 2011; Markman et al., 2010; Seider et al., 2009), e prevenir violência por parceiro íntimo desencadeada por problemas de comunicação (Hammett, Castañeda, \& Ulloa, 2016). 
No Brasil, há escassez de pesquisas sobre comunicação conjugal (Luz \& Mosmann, 2018), inclusive, análises diádicas foram encontradas somente em estudos internacionais (Hammett et al., 2016; Madhyastha et al., 2011; Markman et al., 2010; Seider et al., 2009). Quanto a investigação de casais autodeclarados heterossexuais, há evidências científicas em direções contrárias quanto à correspondência entre as variáveis independentes de maridos e esposas sobre a variável de desfecho ajustamento conjugal de ambos. Alguns estudos sugerem que os homens são mais habilidosos e resolutivos quando se trata da resolução de problemas (Delatorre et al., 2017; Delatorre \& Wagner, 2018; Yaşın \& Sunal, 2016), o que reverbera positivamente no relacionamento. Diferentemente, as mulheres tendem a ser mais empáticas e a expressar com mais facilidade o que sentem (Costa \& Mosmann, 2020; Deitz et al., 2015). Outras pesquisas são inconclusivas quanto ao efeito da variável independente gênero sobre o ajustamento conjugal, ou seja, se é o marido (Iveniuk et al., 2014), ou a esposa (Terveer \& Wood, 2014), quem interfere mais na dinâmica conjugal. Portanto, os estudos revelam diferenças associadas ao gênero e a necessidade de mais evidências cientificas para clarificar se em díades conjugais heterossexuais o fator de impacto ator/parceiro da comunicação sobre ajustamento conjugal apresenta ou não variações.

Soma-se a isso que a apreciação do fenômeno necessita empregar métodos mais consistentes de investigação e avaliação, considerando a comunicação um fenômeno interacional que se retroalimenta (Boscolo \& Bertrando, 2013; Costa et al., 2016; Féres-Carneiro \& DinizNeto, 2010; Mattelart, 2009; Watzlawick et al., 1973). Para tanto, análises diádicas em que se consideram os efeitos ator/parceiro em modelos interdependentes - APIM (Andrade, CasseppBorges, Ferrer, \& Sanchez-Aragón, 2017; Kenny, Kashy, \& Cook, 2006) podem contribuir para uma avaliação mais robusta do impacto que os diferentes tipos de comunicação provocam sobre o ajustamento conjugal.

Por meio do APIM é possível identificar se o estilo de comunicação de um membro da relação impacta no ajustamento conjugal do outro membro (efeito parceiro) e no ajustamento conjugal do próprio indivíduo (efeito ator) (Andrade et al., 2017; Kenny et al., 2006). Ainda, nessas análises é possível avaliar a magnitude do impacto das variáveis independentes sobre as variáveis de desfecho e a força das correlações entre elas.

Com base no exposto, o presente estudo analisou se os estilos de comunicação conjugal negativa e aberta impactam no ajustamento conjugal de casais heterossexuais. Será avaliado o impacto que os estilos de comunicação produzem no ajustamento conjugal de maridos e esposas, considerando as seguintes hipóteses: $\mathrm{H} 1$ = o efeito ator/mulher da comunicação negativa sobre o ajustamento conjugal será superior que o efeito ator/homem; $\mathrm{H} 2=0$ efeito ator/mulher da comunicação aberta sobre o ajustamento conjugal será inferior que o efeito ator/homem; H3 = o efeito parceiro/mulher da comunicação negativa e aberta sobre o ajustamento conjugal será superior que o efeito parceiro/homem.

\section{Materiais e Método}

\section{Delineamento}

O estudo caracteriza abordagem quantitativa de natureza transversal e delineamento correlacional e explicativo. Tentará explicar, a partir de um modelo proposto teoricamente, o porquê do fenômeno investigado, testando-se as hipóteses levantadas (Creswell, 2010).

\section{Participantes}

Os participantes deste estudo foram 231 casais heterossexuais (462 indivíduos). A idade mínima dos respondentes foi de 18 anos e a máxima de 79 anos $(M=41,41 ; D P=12,40)$, e o tempo 
de união variou de 6 meses a 53 anos $(M=15,15 ; D P=12,05)$. Outras informações sociodemográficas da amostra constam na Tabela 1.

Tabela 1

Características sociodemográficas da amostra $(N=462)$

\begin{tabular}{|c|c|c|c|}
\hline & \multirow[t]{2}{*}{ Variáveis sociodemográficas } & \multicolumn{2}{|c|}{ Participantes } \\
\hline & & $n$ & Frequência \% \\
\hline \multirow{5}{*}{ Escolaridade } & Nível fundamental & 37 & 8,0 \\
\hline & Nível médio & 110 & 23,8 \\
\hline & Nível técnico & 28 & 6,1 \\
\hline & Nível superior & 120 & 26 \\
\hline & Pós-graduação lato e stricto sensu & 167 & 36,1 \\
\hline \multirow{3}{*}{$\begin{array}{l}\text { Local de } \\
\text { residência }\end{array}$} & Porto Alegre & 74 & 16 \\
\hline & Região metropolitana & 64 & 13,9 \\
\hline & Cidades do interior do estado & 324 & 70,1 \\
\hline \multirow{4}{*}{$\begin{array}{l}\text { Situação } \\
\text { conjugal }\end{array}$} & Casado somente no civil & 64 & 13,9 \\
\hline & Casado somente no religioso & 11 & 2,4 \\
\hline & Casado no civil e no religioso & 206 & 44,6 \\
\hline & Morando junto - união estável & 181 & 39,2 \\
\hline \multirow{3}{*}{$\mathrm{N}^{\mathrm{o}}$ de filhos } & Sem filhos & 156 & 33,8 \\
\hline & 1 ou 2 filhos & 260 & 56,2 \\
\hline & 3,4 ou 5 filhos & 46 & 10 \\
\hline \multirow{3}{*}{ Trabalho } & Aposentado ou sem trabalho & 75 & 16,2 \\
\hline & Fora de casa & 387 & 83,8 \\
\hline & Horas de trabalho fora de casa & $M=6,67$ & $\mathrm{DP}=3,31$ \\
\hline \multirow{6}{*}{ Renda } & Não tem renda & 38 & 8,2 \\
\hline & Até 1 salário & 31 & 6,7 \\
\hline & De 2 a 3 salários & 167 & 36,1 \\
\hline & De 4 a 6 salários & 120 & 26 \\
\hline & De 7 a 10 salários & 64 & 13,9 \\
\hline & 11 salários ou mais & 42 & 9,1 \\
\hline \multirow{5}{*}{ Religião } & Católica & 313 & 67,7 \\
\hline & Evangélica & 47 & 10,2 \\
\hline & Espírita & 56 & 12,1 \\
\hline & Protestante & 9 & 1,9 \\
\hline & Sem religião & 37 & 8 \\
\hline
\end{tabular}

\section{Instrumento}

Questionário Sociodemográfico: o levantamento das características sociodemográficas dos participantes foi feito por meio de um questionário com quinze questões que investigaram: idade, orientação sexual, local de residência, situação conjugal, tempo de união no relacionamento atual, existência de relacionamento anterior, tempo de união na relação anterior, escolaridade, profissão, trabalho fora de casa, carga horária de trabalho, renda pessoal, número de filhos e religião.

Revised Dyadic Adjustment Scale - R-DAS (Busby, Christensen, Crane, \& Larson, 1995; validado por Hollist et al., 2012). A versão reduzida da escala de ajustamento conjugal possui 14 itens que constituem três fatores. O primeiro, consenso, possui seis itens que avaliam o nível de concordância/discordância entre os parceiros sobre diferentes temas em uma escala Likert de cinco 
pontos que variam de cinco "sempre concordamos" até zero "sempre discordamos". O fator satisfação possui quatro itens que medem a frequência com que os parceiros brigam, conversam sobre divórcios, entre outros temas, em uma escala Likert de cinco pontos que varia de zero "sempre" até cinco "nunca". O terceiro fator, coesão, possui quatro itens que avaliam a frequência com os parceiros realizam diferentes atividades juntos. Os itens devem ser pontuados em uma escala Likert de cinco pontos que variam de zero "nunca" até cinco "mais de uma vez por dia", à exceção do item 11 que é pontuado em uma escala Likert de quatro pontos, sendo quatro "todos os dias" e zero "nunca". Na tradução e validação para o Brasil foram encontrados Alpha de Cronbach de 0,90 para ajustamento total, 0,81 para o fator consenso, 0,85 para satisfação e 0,80 para coesão. Neste estudo, os valores do Alpha de Cronbach para ajustamento total e fatores consenso, satisfação e coesão, foram, respectivamente, $0,84,0,77,0,78$ e 0,80 para os homens e $0,87,0,72,0,83$ e 0,82 para as mulheres.

Communication Questionnaire (Van den Troost et al., 2005; traduzido por Luz \& Mosmann, 2018). Trata-se de uma escala composta por quinze itens divididos em dois fatores. $\mathrm{O}$ primeiro possui nove itens que avaliam a comunicação negativa e o segundo possui seis itens que avaliam a comunicação aberta. $\mathrm{O}$ respondente pontua em uma escala Likert de sete pontos que variam de um "não aplicável" até sete "muito aplicável", o quanto cada afirmação corresponde a forma como os parceiros conjugais se comunicam. No estudo brasileiro de adaptação da escala foi encontrado Alpha de Cronbach de 0,74 para comunicação negativa e de 0,70 para comunicação aberta (Luz \& Mosmann, 2018). No estudo original, foi encontrado para homens e mulheres, respectivamente, Alpha de Cronbach de 0,83 e 0,80 no fator comunicação negativa e 0,67 e 0,71 no fator comunicação aberta. Neste estudo, os valores do Alpha de Cronbach para os fatores comunicação negativa e comunicação aberta foram, respectivamente 0,86 e 0,81 para os homens e 0,83 e 0,83 para as mulheres.

\section{Procedimentos de Coleta de Dados}

A coleta de dados ocorreu em Porto Alegre, região metropolitana e cidades do interior do estado do Rio Grande do Sul a fim de que a amostra fosse heterogênea. O pesquisador responsável entrou em contato com os casais via telefone, WhatsApp e e-mail por meio da indicação de pessoas conhecidas, portanto, coleta de dados por conveniência. No primeiro contato foram explicados os objetivos do estudo, os riscos e benefícios implicados na participação. Havendo interesse e disponibilidade, agendava-se dia e hora para a coleta no local de preferência do casal, este que variou entre residência e local de trabalho. O procedimento levou em média 60 minutos e envolveu a leitura em voz alta do Termo de Consentimento Livre e Esclarecido - TCLE, esclarecimento de dúvidas, assinatura do termo em quatro vias, cada membro do casal ficava com uma via e devolvia a outra para o pesquisador que guardaria os documentos separadamente dos questionários, evitando a identificação dos participantes com base no TCLE. Por fim, realizou-se o preenchimento do questionário de pesquisa, este que foi realizado pelo casal separadamente, ou seja, sem que um tivesse acesso as respostas do outro. Além disso, cada questionário de pesquisa estava identificado com a letra correspondente ao sexo, homem " $\mathrm{H}$ " e mulher " $\mathrm{M}$ " e um número correspondente à díade, conforme exemplo: Envelope 1 - Questionários H1 e M1; Envelope 2 Questionários H2 e M2, etc.

\section{Considerações Éticas}

O presente estudo foi submetido à avaliação do Comitê de Ética em Pesquisa da Universidade do Vale do Rio dos Sinos, UNISINOS. Os procedimentos adotados seguiram rigorosamente o que consta na Resolução 510/2016 do Conselho Nacional de Saúde, atendendo os fundamentos éticos e científicos pertinentes, conforme lia-se no TCLE. Entre as informações fornecidas no termo constava: participação livre e voluntária na pesquisa, possibilidade de 
desistência sem prejuízos, riscos e benefícios, garantia de sigilo e proteção das informações, direito à solicitação dos resultados do estudo, assistência e encaminhamento em caso de dano psicológico devido a participação na pesquisa e contato dos pesquisadores responsáveis.

\section{Análise dos Dados}

Inicialmente foi construído um banco de dados por meio do programa SPSS 25.0 (Statistical Package for Social Science), comprovados os critérios de normalidade da amostra e então realizadas análises descritivas para calcular porcentagens, médias e desvio padrão. Foi construído também um segundo banco de dados organizado em estrutura própria para a realização de análises diádicas. Nesse formato, diferente do usual em que cada indivíduo corresponde a uma linha do bando de dados, cada casal corresponde a uma linha do banco. Há, portanto, o dobro de variáveis e a redução da amostra pela metade (Andrade et al., 2017). Para a realização das análises Actor-Partner Interdependence Model - APIM foi utilizado o programa AMOS 22.0 (Analysis of Moment Structures) no qual se realiza a Structural Equation Modeling - SEM (Modelagem de Equação Estrutural - MEE), técnica multivariada de análise de dados em que é possível testar relações simultâneas entre variáveis dependentes e independentes por meio de modelos teóricos de relações múltiplas (Byrne, 2010; Pilati \& Laros, 2007).

O primeiro passo da análise foi a verificação da interdependência ou não-independência entre os membros da díade, já que em relacionamentos românticos é esperado que exista um contexto compartilhado e, portanto, influência recíproca entre os parceiros. A presença de interdependência possibilita indicar a influência entre a díade e incluir como unidade de análise tanto o indivíduo quanto o casal. Considerando, em modelos diádicos, o emparelhamento das duplas, a interdependência foi avaliada por meio do coeficiente de correlação de Pearson das respostas para um mesmo conjunto de variáveis (Kenny et al., 2006).

No segundo passo, utilizando o banco organizado em estrutura individual, foram realizadas as Análises Fatoriais Confirmatórias Multigrupo para verificar a invariância de parâmetros entre os grupos de homens e mulheres, ou seja, se os construtos avaliados são os mesmos para ambos os membros da díade. Por meio do teste de invariância é possível inferir que os itens que compõem determinado fator apresentam a mesma carga fatorial para os dois grupos (Damásio, 2013). Segundo Byrne (2010), para se assumir a invariância entre os grupos é aceitável uma diferença de no máximo 0,010 no índice de ajuste CFI dos modelos.

No terceiro passo, o modelo APIM proposto teoricamente foi testado verificando-se os efeitos da comunicação negativa e da comunicação aberta sobre o ajustamento conjugal, considerando os efeitos ator/homem e ator/mulher e os efeitos parceiro/homem e parceiro/mulher. Inicialmente foram calculadas as médias de cada um dos fatores avaliados por meio do somatório dos itens e, posteriormente, testado o modelo. Foram utilizados os seguintes índices de ajuste do modelo: Qui-quadrado $\left(\chi^{2}\right)$, Comparative Fit Index (CFI), Non-Normed Fit Index (NNFI) e Root Mean Square Error of Approximation (RMSEA) com intervalo de confiança de $90 \%$. O método de estimação foi o de máxima verossimilhança (Maximum Likelihood - ML). Valores menores de Qui-quadrado, superiores a 0,900 para CFI e NNFI e inferiores a 0,080 para o RMSEA são aceitáveis e indicam bom ajuste do modelo. Sugere-se, preferencialmente, índices de ajuste superiores a 0,950 para CFI e NNFI e inferiores a 0,050 para o RMSEA (Byrne, 2010).

\section{Resultados}

Por meio dos testes iniciais foi possível inferir a interdependência entre os membros da díade, conforme pode ser observado na Tabela 2 em que são apresentadas médias, desvios padrão e correlações entre as escalas de comunicação e o R-DAS para homens e mulheres. 
Tabela 2.

Comunicação e Ajustamento Conjugal: médias, desvios padrão e correlações $(N=462)$

\begin{tabular}{|c|c|c|c|c|c|}
\hline \multirow[b]{2}{*}{ Média } & \multirow{2}{*}{$\begin{array}{l}\text { Desvio } \\
\text { padrão }\end{array}$} & \multirow[b]{2}{*}{$\begin{array}{l}\text { Variáveis da } \\
\text { mulher }\end{array}$} & \multicolumn{3}{|c|}{ Variáveis do homem } \\
\hline & & & Comunicação negativa & $\begin{array}{c}\text { Comunicação } \\
\text { aberta }\end{array}$ & $\begin{array}{l}\text { Ajustamento } \\
\text { conjugal }\end{array}$ \\
\hline 24,13 & 9,439 & $\begin{array}{c}\text { Comunicação } \\
\text { negativa }\end{array}$ & $495^{* *}$ &,$- 233^{* *}$ &,$- 494^{* *}$ \\
\hline 26,50 & 7,437 & $\begin{array}{c}\text { Comunicação } \\
\text { aberta }\end{array}$ &,$- 175^{* *}$ &, $388^{* *}$ &, $304^{* *}$ \\
\hline 50,12 & 9,046 & $\begin{array}{l}\text { Ajustamento } \\
\text { conjugal }\end{array}$ &,$- 478^{* *}$ &, $306^{* *}$ &, $615^{* *}$ \\
\hline
\end{tabular}

O teste de invariância das cargas fatoriais para os dois grupos é apresentado na Tabela 3. Ao comparar a invariância configural no modelo 0 sem restrição e a invariância métrica no modelo 1 com restrição das cargas fatoriais verifica-se que a diferença no índice de comparação de ajuste (Comparative Fit Index-CFI), não foi superior a 0,010 para nenhum dos fatores.

Tabela 3

Fatorial Multigrupo das Escalas de Comunicação e do Ajustamento Conjugal de Homens e Mulheres $(N=462)$

\begin{tabular}{|c|c|c|c|c|c|c|}
\hline Fator & $\begin{array}{l}\text { Comparação } \\
\text { dos } \\
\text { modelos }\end{array}$ & $\chi^{2}$ & $d f$ & $p$ & CFI & RMSEA (90\%) \\
\hline \multirow{3}{*}{$\begin{array}{c}\text { Comunicação } \\
\text { negativa }\end{array}$} & Modelo 0 & 80,673 & 48 & $<0,002$ & 0,979 & 0,038 \\
\hline & $\begin{array}{l}\text { Invariância } \\
\text { configural }\end{array}$ & & & & & $(0,023-0,053)$ \\
\hline & $\begin{array}{l}\text { Modelo } 1 \\
\text { Invariância } \\
\text { métrica }\end{array}$ & 99,940 & 56 & $<0,000$ & 0,972 & $\begin{array}{c}0,041 \\
(0,028-0,054)\end{array}$ \\
\hline \multirow{3}{*}{$\begin{array}{c}\text { Comunicação } \\
\text { aberta }\end{array}$} & Modelo 0 & 31,601 & 16 & $<0,011$ & 0,987 & 0,046 \\
\hline & $\begin{array}{l}\text { Invariância } \\
\text { configural }\end{array}$ & & & & & $(0,021-0,070)$ \\
\hline & $\begin{array}{l}\text { Modelo } 1 \\
\text { Invariância } \\
\text { métrica }\end{array}$ & 35,519 & 21 & $<0,025$ & 0,988 & $\begin{array}{c}0,039 \\
(0,014-0,060)\end{array}$ \\
\hline \multirow{3}{*}{$\begin{array}{l}\text { Ajustamento } \\
\text { conjugal }\end{array}$} & Modelo 0 & 236,114 & 134 & $<0,000$ & 0,955 & 0,041 \\
\hline & $\begin{array}{l}\text { Invariância } \\
\text { configural }\end{array}$ & & & & & $(0,032-0,049)$ \\
\hline & $\begin{array}{l}\text { Modelo } 1 \\
\text { Invariância } \\
\text { métrica }\end{array}$ & 259,676 & 147 & $<0,000$ & 0,951 & $\begin{array}{c}0,041 \\
(0,033-0,049)\end{array}$ \\
\hline
\end{tabular}

Os testes de interdependência e a Análise Fatorial Confirmatória Multigrupo demonstraram ser possível prosseguir à execução da análise diádica pelo método Actor Partner Interdependence Model - APIM. O modelo final, representado graficamente na Figura 1, apresentou bons índices de ajuste, sendo possível avaliar o impacto da comunicação negativa e aberta sobre o ajustamento conjugal dos membros da díade. Os índices de ajuste do modelo foram: $[\chi 2=21,326 ; d f=4$; $p=0,000 ; \mathrm{CFI}=0,965, \mathrm{NNFI}=0,958 ; \mathrm{RMSEA}=0,092(90 \% \mathrm{CI}=0,084-0,137)]$. 


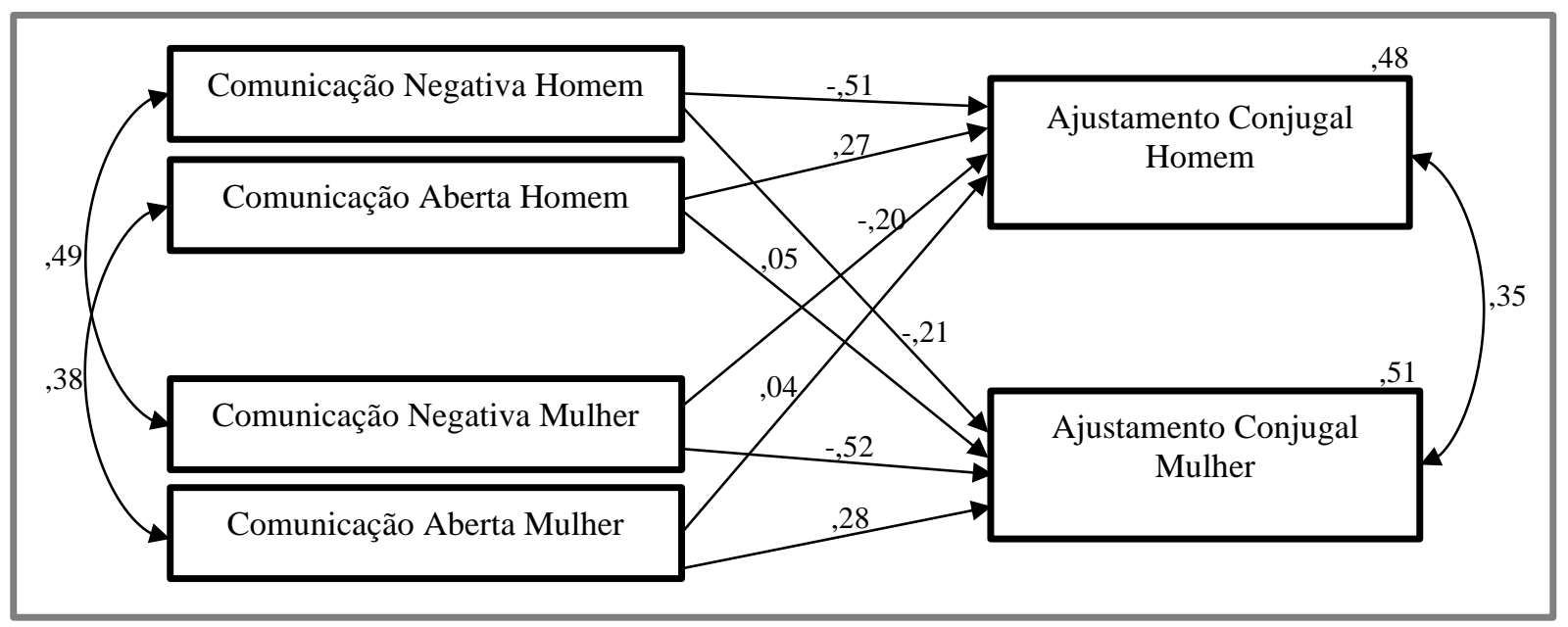

Figura 1.

Modelo APIM para comunicação e ajustamento conjugal

Além dos índices de ajuste, o modelo APIM permite estimar a covariância entre as variáveis independentes e entre os erros das variáveis dependentes ou de desfecho de homens e mulheres (Andrade et al., 2017). No fator comunicação negativa verificou-se covariância moderada $(r=0,49)$, na comunicação aberta covariância leve $(r=0,38)$, e entre os erros da variável de desfecho ajustamento conjugal covariância leve $(r=0,35)$.

A magnitude de predição da regressão indicando o impacto do efeito ator (variáveis independentes do indivíduo interferindo no seu próprio ajustamento conjugal) e do efeito parceiro (variáveis independentes de um membro da relação impactando no ajustamento do cônjuge), podem ser observadas por meio das setas que partem da esquerda para a direita na Figura 1. Verifica-se que o efeito ator/homem da comunicação negativa e da comunicação aberta sobre o ajustamento conjugal foi de $(B=-0,51 ; p=0,001)$ e $(B=0,27 ; p=0,001)$, respectivamente, e o efeito ator/mulher dos mesmos fatores sobre o ajustamento conjugal foi de $(B=-0,52 ; p=0,001)$ e $(B=0,28 ; p=0,001)$.

Finalmente, o efeito parceiro/homem dos estilos de comunicação negativa e aberta sobre o ajustamento conjugal, ou seja, o impacto que a forma de se comunicar do marido provoca sobre o ajustamento conjugal da esposa, foi de $(B=-0,21 ; p=0,001)$ e $(B=0,05 ; p=0,227)$, respectivamente. $\mathrm{O}$ efeito parceiro/mulher dos estilos de comunicação negativa e aberta sobre o ajustamento conjugal do marido foi de $(B=-0,20 ; p=0,001)$ e $(B=0,04 ; p=0,490)$. Observa-se, nesses resultados, que o efeito ator/homem e o efeito ator/mulher da comunicação negativa sobre o ajustamento conjugal foi moderado e da comunicação aberta leve. Analisando-se o efeito parceiro/homem e o efeito parceiro/mulher da comunicação sobre o ajustamento conjugal, verifica-se magnitude leve para comunicação negativa e ausência de predição para comunicação aberta, considerado o valor de $p<0,05$.

\section{Discussão}

O objetivo do presente estudo foi analisar se os estilos de comunicação negativa e aberta impactam no ajustamento conjugal de casais heterossexuais. Os resultados encontrados por meio do modelo Actor Partner Interdependence Model - APIM, possibilitam compreender 
características relevantes da interação entre homens e mulheres que vivem em relacionamentos conjugais heterossexuais (Madhyastha et al., 2011; Markman et al., 2010; Seider et al., 2009).

$\mathrm{O}$ primeiro aspecto elucidado se refere à associação entre a forma de se comunicar dos casais. Tal se observa por meio da covariância entre o estilo de comunicação negativa do marido e da esposa, da mesma forma como ocorre com o estilo de comunicação aberta e com o ajustamento conjugal, covariância entre os erros. O resultado dessas covariâncias indica que se ocorre alteração na forma de se comunicar, negativa e/ou aberta, e no ajustamento conjugal de um membro da díade, a mudança provocará alteração diretamente proporcional na forma de se comunicar e no ajustamento conjugal do parceiro.

Conforme referido na introdução, é necessário investigar os aspectos interacionais ao se trabalhar com casais, já que o foco principal são as características que marcam a forma como os parceiros se relacionam e desenvolvem padrões de interação que tendem a se repetir (Costa et al., 2016; Féres-Carneiro \& Diniz-Neto, 2010). O resultado indica a interdependência entre os membros da díade, ou seja, a forma de se comunicar de um parceiro interfere na forma de se comunicar do outro, seja negativa ou positivamente (comunicação aberta), influência considerada necessária por estudiosos do fenômeno (Driver et al., 2016; Madhyastha et al., 2011).

A interdependência pode ser observada também no ajustamento conjugal. Em uma perspectiva clínica, esse resultado poderia indicar que é imprescindível avaliar o contexto relacional em que ocorrem os problemas e os conflitos conjugais já que um parceiro tende a ser um termômetro do que ocorre com o outro. Além disso, esse aspecto corrobora o pressuposto sistêmico de retroalimentação (causalidade circular), realçando a necessidade de consideração das características da comunicação ao se trabalhar com casais (Boscolo \& Bertrando, 2013; Mattelart, 2009; Watzlawick et al., 1973). Outra compreensão refere-se ao caráter complementar da comunicação. Conforme postulado por Watzlawick et al. (1973), não é correto supor que um provoca a posição do outro de forma linear, mas que ambos mutuamente criam oportunidades para que isto se estabeleça e se mantenha. Nesse sentido comunicação negativa gera mais interações negativas e vice-versa.

Os resultados da análise do efeito ator sobre o ajustamento conjugal indicaram que a forma de comunicar dos parceiros impacta o ajustamento do próprio indivíduo. Destaca-se que o impacto da comunicação negativa foi superior ao impacto da comunicação aberta sobre o ajustamento de homens e mulheres. Essa evidência indica que o estilo de comunicação de um indivíduo interfere nos seus níveis de ajustamento conjugal e, principalmente, que a comunicação negativa tende a impactar mais, corroborando outros estudos (Johnson et al., 2005; Markman et al., 2010) que atestam a superioridade das questões negativas como fatores de risco em comparação às questões positivas enquanto fatores de proteção.

Os efeitos parceiro/homem e parceiro/mulher das variáveis independentes comunicação negativa e aberta sobre as variáveis de desfecho ajustamento conjugal suscitam importantes reflexões, já que apenas o estilo de comunicação negativa impactou o ajustamento conjugal, resultado equivalente para homens e mulheres. É possível encontrar embasamento para esse resultado nos estudos conduzidos pelos pesquisadores do laboratório Gottman (Driver et al., 2016). Segundo eles, casais satisfeitos tendem a ter cinco interações positivas para cada interação negativa se comparados com casais insatisfeitos ou que se divorciam, que não conseguem desenvolver a mesma equação ao interagir. Portanto, pode-se conjecturar por meio desse resultado que para impactar no ajustamento conjugal os níveis de comunicação positiva (aberta) deveriam ser cinco vezes superiores aos níveis de comunicação negativa, o que pode explicar a ausência de predição desta última variável sobre o ajustamento conjugal.

Além do efeito parceiro ocorrer apenas para o estilo de comunicação negativa, ao considerar os resultados do efeito ator sobre o ajustamento conjugal se verifica o impacto superior da comunicação negativa. Em conjunto, esses dados confirmam pressupostos de renomados pesquisadores investigadores do fenômeno (Watzlawick et al., 1973), de que a comunicação torna- 
se um elemento secundário se for espontânea (positiva), entretanto, em contextos de interação conjugal conflituosos, o estilo de comunicação fica em evidência, compreensão sustentada também por estudos empíricos que indicam a preponderância das dimensões negativas sobre as positivas nas relações conjugais (Johnson et al., 2005; Luz \& Mosmann, 2018; Markman et al., 2010).

Os resultados do efeito ator e parceiro foram preponderantes em relação à comunicação negativa, confirmando pesquisas anteriores sobre o impacto que essa forma de se comunicar provoca na conjugalidade (Costa et al., 2017; Johnson et al., 2005; Luz \& Mosmann, 2018; Markman et al., 2010). Essa evidência pode indicar que os esforços empreendidos nos programas de educação conjugal e nas intervenções clínicas com casais deveriam focalizar principalmente na redução da comunicação negativa e das características desse tipo de comunicação (Van den Troost et al., 2005), e menos em promover habilidades de comunicação positiva entre os parceiros, compreensão divergente do que foi referido por Baucom et al. (2015) de que a comunicação construtiva proporcionaria efeitos mais consistentes em terapia de casal do que a comunicação destrutiva.

Finalmente, as hipóteses de que existiriam diferenças entre maridos e esposas quanto ao efeito ator/parceiro da comunicação negativa e aberta sobre o ajustamento conjugal dos cônjuges não foram confirmadas. Diferente do que foi encontrado em outros estudos (Costa \& Mosmann, 2020; Deitz et al., 2015; Iveniuk et al., 2014; Terveer \& Wood, 2014), verificou-se similaridade de impacto das variáveis independentes de maridos e esposas sobre o ajustamento de ambos. Em síntese, o resultado levanta dúvidas acerca da efetiva diferença entre homens e mulheres quando estes se envolvem em um relacionamento conjugal estável e passam a se influenciar mutuamente, de tal forma que se torna difícil determinar como se estabelecem os processos comunicacionais e seus efeitos sobre o ajustamento conjugal que novamente impacta a forma de se comunicar. Em uma perspectiva sistêmica essa determinação é irrelevante, principalmente porque há o pressuposto de causalidade circular e de que a retroalimentação dentro de um sistema só ocorre porque as partes - interlocutores, contribuem para a manutenção do circuito interacional (Boscolo \& Bertrando, 2013; Mattelart, 2009; Watzlawick et al., 1973). Novamente, é relevante considerar a complementariedade nos processos comunicacionais, ainda que aparentemente existam diferenças, elas se ajustam para a manutenção da dinâmica conjugal - enquanto o gênero não é relevante neste aspecto, mas sim o papel desempenhado por cada cônjuge nas interações.

\section{Conclusão}

Os estilos de comunicação investigados nesse estudo e o impacto que provocam sobre o ajustamento conjugal foram evidenciados por meio de análises diádicas, analisando-se o efeito da forma de se comunicar do indivíduo sobre ele mesmo e sobre o parceiro. Foram encontradas evidências de que a compreensão do fenômeno necessita ocorrer em uma perspectiva sistêmica sobretudo porque, nas relações conjugais, verifica-se níveis significativos de interdependência entre os parceiros, ou seja, o que ocorre com um tende a influenciar o mesmo aspecto no outro.

Nas pesquisas com casais é fundamental a realização de coleta diádica de dados que permite analisar a influência entre os parceiros, já que o fenômeno envolve uma dupla que vive em um mesmo contexto, compartilha interesses e projetos. Por outro lado, coletar presencialmente dados de casais envolve expressivo tempo e dedicação dos pesquisadores e grupos de pesquisa, o que se torna um aspecto desafiador na condução de investigações dessa natureza. Um ponto forte do presente estudo é justamente o trabalho com díades e a realização de análises que permitiram avaliar o fenômeno em uma perspectiva mais abrangente, considerando a interação entre os parceiros.

Por outro lado, o fato de ser uma pesquisa conduzida por meio de escalas de autorrelato, em que o participante responde sobre si e sobre o parceiro, pode ser considerada uma limitação 
deste estudo. Embora exista o pressuposto de que parceiros românticos se conhecem e, portanto, estão aptos a inferir sobre a percepção do cônjuge, estudos experimentais e longitudinais são uma alternativa para minimizar essa limitação deixada pelos estudos transversais e que utilizam escalas de autorrelato.

Além disso, estudos transversais apresentam outras limitações, como por exemplo, análise da amostra com base em um momento específico no tempo, já que as respostas são emitidas com base em uma avaliação restrita à memória recente dos respondentes, está que considerará eventos e experiências vividas mais recentemente no relacionamento. Quanto a ser uma amostra por conveniência, pode ocorrer de as pessoas selecionadas pertencerem a um nicho social excessivamente homogêneo, neste estudo, participantes predominantemente católicos, com filhos, pertencentes à classe média e com alto nível de escolarização. Portanto, os resultados e as reflexões propostas representam, em certa medida, um grupo social com tais características sociodemográficas.

Nos resultados deste estudo, o estilo de comunicação negativa se destacou pela força das correlações entre a díade, pela magnitude de predição enquanto efeito ator/homem e efeito ator/mulher e, principalmente, porque no efeito parceiro/homem e parceiro/mulher foi o único fator de predição. Esses resultados sugerem uma agenda de pesquisas sobre os relacionamentos românticos que deve atentar para a interdependência e retroalimentação dos fatores individuais e diádicos e avaliar se, conforme encontrado neste estudo, as questões negativas provocam impactos mais robustos na conjugalidade, orientando, assim, programas de prevenção e educação conjugal para atender esse tipo de demanda dos casais, especialmente em relação à manutenção ao longo do tempo de casamento.

Ainda, esse resultado tem relevantes implicações para a área clínica, pois indica a necessidade de os terapeutas de casal focalizarem suas intervenções na diminuição da comunicação negativa e do contexto de negatividade que se desenvolve se a comunicação entre os cônjuges é disfuncional, ao invés de focalizar na promoção de comportamentos positivos. Ademais, intervir na interação e, consequentemente, nos mecanismos de retroalimentação entre os parceiros, pode ser o método mais eficaz se o foco são os problemas conjugais, já que os dados comprovam que a alteração de qualquer uma das variáveis provocará impacto no parceiro e na relação.

\section{Referências}

Andrade, A. L., Cassepp-Borges, V., Ferrer, E., \& Sanchez-Aragón, R. (2017). Análises de dados diádicos: Um exemplo a partir da pesquisa com casais. Temas em Psicologia, 25(4), 15711588. doi: 10.9788/TP2017.4-05

Baucom, K. J. W., Baucom, B. R., \& Christensen, A. (2015). Changes in dyadic communication during and after integrative and traditional behavioral couple therapy. Behavior Research and Therapy, 65, 18-28. doi: 10.1016/j.brat.2014.12.004

Belanger, C., Laporte, L., Sabourin, S., \& Wright, J. (2015). The effect of cognitive-behavioral group marital therapy on marital happiness and problem-solving self-appraisal. The American Journal of Family Therapy, 43(2), 103-118. doi: 10.1080/01926187.2014.956614

Blanchard, V. L., Hawkins, A. J., Baldwin, S. A., \& Fawcett, E. B. (2009). Investigating the effects of marriage and relationship education on couples' communication skills: A meta-analytic study. Journal of Family Psychology, 23(2), 203-214. doi: 10.1037/a0015211

Boscolo, L., \& Bertrando, P. (2013). Terapia sistêmica individual: Manual prático na clínica. Belo Horizonte: Artesã.

Busby, D. M., Christensen, C., Crane, D. R., \& Larson, J. H. (1995). A revision of the Dyadic Adjustment Scale for use with distressed and nondistressed couples: Construct hierarchy and 
multidimensional scales. Journal of Marital and Family Therapy, 21(3), 289-308. doi: 10.1111/j.17520606.1995.tb00163.x

Byrne, B. M. (2010). Structural equation modeling with AMOS: Basic concepts, applications, and programming ( $2^{\text {nd }}$ ed.). New York: Routledge.

Costa, C. B., Cenci, C. B., \& Mosmann, C. P. (2016). Conflitos conjugais e estratégias de resolução: Uma revisão sistemática da literatura. Temas em Psicologia, 24(1), 1-14, doi: 10.9788/TP2016.1-22

Costa, C. B., Delatorre, M. Z., Wagner, A., \& Mosmann, C. P. (2017). Terapia de casal e estratégias de resolução de conflito: Uma revisão sistemática. Psicologia: Ciência e Profissão, 37(1), 208-223. doi: 10.1590/1982-3703000622016

Costa, C. B., \& Mosmann, C. P. (2015). Estratégias de resolução dos conflitos conjugais: Percepções de um grupo focal. Revista Psico, 46(4), 472-482. doi: 10.15448/19808623.2015.4.20606

Costa, C. B., \& Mosmann, C. P. (2020). Aspects of the marital relationship that characterize secure and insecure attachment in men and women. Estudos de Psicologia (Campinas), 37, e190045. doi: 10.1590/1982275202037e190045

Creswell, J. W. (2010). Projeto de pesquisa: Métodos qualitativo, quantitativo e misto. 3. ed. Porto Alegre: Artmed.

Damásio, B. F. (2013)._Contribuições da Análise Fatorial Confirmatória Multigrupo (AFCMG) na avaliação de invariância de instrumentos psicométricos. Psico-USF, 18(2), 211-220.

Deitz, S. L., Anderson, J. R., Johnson, M. D., Hardy, N. R., Zheng, F., \& Liu, W. (2015). Young romance in China: Effects of family, attachment, relationship confidence, and problem solving. Personal Relationships, 22(2), 243-258. doi: 10.1111/pere.12077

Delatorre, M. Z., Scheeren, P., \& Wagner, A. (2017). Conflito conjugal: Evidências de validade de uma escala de resolução de conflitos em casais do sul do Brasil. Avances en Psicología Latinoamericana, 35(1), 79-94. doi: 10.12804/revistas.urosario.edu.co/apl/a.3742

Delatorre, M. Z., \& Wagner, A. (2018). Marital conflict management of married men and women. Psico-USF, 23(2), 229-240. doi: 10.1590/141382712018230204

Driver, J., Tabares, A., Shapiro, A. F., \& Gottman, J. M. (2016). Interação do casal em casamentos com altos e baixos níveis de satisfação. Estudos do Laboratório Gottman. In F. Walsh (Org.), Processos normativos da família: Diversidade e complexidade (S. M. M. Rosa, Trad., $4^{\text {th }}$ ed.), (pp. 57-77). Porto Alegre: Artmed.

Epstein, R., Warfel, R., Johnson, J., Smith, R., \& McKinney, P. (2013). Which relationship skills count most? Journal of Couple \& Relationship Therapy, 12(4), 297-313. doi: $10.1080 / 15332691.2013 .836047$

Féres-Carneiro, T., \& Diniz-Neto, O. (2008). Psicoterapia de casal: Modelos e perspectivas. Aletheia, 27(1), 173-187.

Hammett, J. F., Castañeda, D. M. \& Ulloa, E. C. (2016). The association between affective and problem-solving communication and intimate partner violence among Caucasian and Mexican American couples: A dyadic approach. Journal of Family Violence, 31(2), 167178. doi: 10.1007/s10896-015-9762-2

Holley, S. R., Haase, C. M., \& Levenson, R. W. (2013). Age-related changes in demand-withdraw communication behaviors. Journal of Marriage and Family, 75(4), 822-836.

Hollist, C. S., Falceto, O. G., Ferreira, L. M., Miller, R. B., Springer, P. R., Fernandes, C. L. C., \& Nunes, N. A. (2012). Portuguese translation and validation of the Revised Dyadic Adjustment Scale. Journal of Marital and Family Therapy, 38(s1), 348-358. doi: 10.1111/j.1752-0606.2012.00296.x

Iveniuk, J., Waite, L. J., Laumann, E., McClintock, M. K., \& Tiedt, A. D. (2014). Marital conflict in older couples: Positivity, personality, and health. Journal of Marriage and Family, 76(1), 130-144. doi: 10.1111/jomf.12085 
Johnson, M. D., Cohan, C. L., Davila, J., Lawrence, E., Rogge, R. D., Karney, B. R., ... \& Bradbury, T. N. (2005). Problem-solving skills and affective expressions as predictors of change in marital satisfaction. Journal of Consulting and Clinical Psychology, 73(1), 15-27. doi: 10.1037/0022006X.73.1.15

Kenny, D. A., Kashy, D. A., \& Cook, W. L. (2006). Dyadic data analysis. New York: The Guilford Press.

Lau, K. K. H., Tao, C., Randall, A. K., \& Bodenmann, G. (2016). Coping-oriented couple therapy. In: Lebow J., Chambers A., Breunlin D. (eds). Encyclopedia of Couple and Family Therapy. Springer, Cham.

Luz, S. K., \& Mosmann, C. P. (2018). Funcionalidade e comunicação conjugal em diferentes etapas do ciclo de vida. Revista da SPAGESP, 19(1), 21-34.

Markman, H. J., Rhoades, G. K., Stanley, S. M., Ragan, E. P., \& Whitton, S. W. (2010). The premarital communication roots of marital distress and divorce: The first five years of marriage. Journal of Family Psychology, 24(3), 289-298. doi: 10.1037/a0019481

Mattelart, A. E. (2009). História das teorias da comunicação (12a ed.). São Paulo: Loyola.

Neumann, A. P., \& Wagner, A. (2017). Reverberations of a Marital Education Program: The moderator's perception. Paidéia, 27(1), 466-474. doi: 10.1590/1982-432727s1201712

Neumann, A. P., Wagner, A., \& Remor, E. (2018). Couple relationship Education Program 'Living as Partners': Evaluation of effects on marital quality and conflict. Psicologia: Reflexão $e$ Crítica, 31(26), 1-13. doi: 10.1186/s41155-018-0106-Z

Paleari, F. G., Regalia, C., \& Fincham, F. D. (2010). Forgiveness and conflict resolution in close relationships: Within and cross partner effects. Universitas Psychologica, 9(1), 35-56.

Pilati, R., \& Laros, J. A. (2007). Modelos de equações estruturais em psicologia: Conceitos e aplicações. Psicologia: Teoria e Pesquisa, 23(2), 205-216.

Rech, B. C. S., Silva, I. M., \& Lopes, R. C. S. (2013). Repercussões do câncer infantil sobre a relação conjugal. Psicologia: Teoria e Prática, 29(3), 257-265.

Seider, B. H., Hirschberger, G., Nelson, K. L., \& Levenson, R. W. (2009). We can work it out: Age differences in relational pronouns, physiology, and behavior in marital conflict. Psychology and Aging, 24(3), 604-613. doi: 10.1037/a0016950

Terveer, A. M., \& Wood, N. D. (2014). Dispositional optimism and marital adjustment. Contemporary Family Therapy: An International Journal, 36(3), 351-362. doi: 10.1007/s10591-013-9292-0

Tilden, T., Hoffart, A., Sexton, H., Finset, A., \& Gude, T. (2011). The role of specific and common process variables in residential couple therapy. Journal of Couple \& Relationship Therapy, 10(3), 262-278. doi: 10.1080/15332691.2011.588100

Van den Troost, A., Vermulst, A., Gerris, J. R. M., \& Matthijs, K. (2005). The Dutch Marital Satisfaction and Communication Questionnaire: A validation study. Psychologica Belgica, 45(3), 185-206. doi: 10.5334/pb-45-3-185

Watzlawick, P., Beavin, J. H., \& Jackson, D. D. (1973). Pragmática da comunicação humana. Um estudo dos padrões, patologias e paradoxos da interação. São Paulo: Cultrix.

Worthington Jr., E. L., Berry, J. W., Hook, J. N., Davis, D. E., Scherer, M., \& Griffin, B. J., et al. (2015). Forgiveness reconciliation and communication conflict resolution interventions versus retested controls in early-married couples. Journal of Counseling Psychology, 62(1), 14-27. doi: $10.1037 /$ cou0000045

Yaşın, F., \& Sunal, A. B. (2016). The mediator role of relationship satisfaction on the relation between time perspective and responses to romantic relationship dissatisfaction. Turkish Journal of Psychology, 31(78), 91-94. 
Financiamento: Este artigo faz parte de um estudo maior proveniente da tese de doutorado do primeiro autor sob orientação da segunda autora e contou com o apoio da Coordenação de Aperfeiçoamento de Pessoal de Nível Superior, CAPES, Brasil. Código de financiamento 001.

Participação dos autores: a) Planejamento e concepção do trabalho; b) Coleta de dados; c) Análise e interpretação de dados; d) Redação do manuscrito; e) Revisão crítica do manuscrito.

C.B.C. contribuiu em a,b,c,d,e; C.P.M. em a,b,c,d,e.

Editora científica responsável: Dra. Cecilia Cracco 